\title{
Role of IncRNA-ATB in ovarian cancer and its mechanisms of action
}

\author{
DONGLAN YUAN ${ }^{1 *}$, XIAOFANG ZHANG ${ }^{2 *}$, YINLING ZHAO $^{1}$, HUA QIAN $^{1}$, HEZHU WANG $^{1}$,

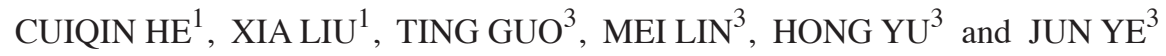 \\ ${ }^{1}$ Department of Gynaecology and Obstetrics, Taizhou People's Hospital, Taizhou, Jiangsu 225300; \\ ${ }^{2}$ Department of Pathology, Jiangxi Provincial Tumor Hospital, Nanchang, Jiangxi 330029; \\ ${ }^{3}$ Translational Medicine Center, Taizhou People's Hospital, Taizhou, Jiangsu 225300, P.R. China
}

Received November 30, 2018; Accepted August 7, 2019

DOI: $10.3892 /$ etm.2019.8282

\begin{abstract}
This study aimed to elucidate the role of long non-coding RNA activated by transforming growth factor- $\beta$ (lncRNA-ATB) in ovarian cancer and its underlying mechanisms of action. Expression levels of lncRNA-ATB in ovarian cancer cell line SKOV3 and in a healthy human ovarian cell line were compared using reverse transcription-quantitative polymerase chain reaction (RT-qPCR). The results indicated that lncRNA-ATB was expressed at significantly higher levels in SKOV3 cells compared with the healthy cell line. After downregulation of 1ncRNA-ATB expression in SKOV3 cells using lncRNA-ATB-short hairpin RNA, cell proliferation, apoptosis, invasion and migration were assessed using Cell counting kit-8, Live Dead staining, Transwell assay and wound healing assay, respectively. RT-qPCR and western blotting were used to quantify the expression of signal transducer and activator of transcription 3 (STAT3), phosphorylated (p)-STAT3, and the additional epithelial to mesenchymal transition (EMT)-related proteins E-cadherin and vimentin in SKOV3 cells. LncRNA-ATB downregulation significantly reduced SKOV3 cell proliferation, invasion and migration, promoted apoptosis, decreased the expression of p-STAT3 and vimentin, and increased E-cadherin expression. Taken together, these results suggest that IncRNA-ATB downregulation can inhibit ovarian cancer cell proliferation, invasion and migration, and promote cell apoptosis. Lnc-RNA-ATB may therefore be a new target for ovarian cancer treatment.
\end{abstract}

Correspondence to: Dr Jun Ye or Dr Hong Yu, Translational Medicine Center, Taizhou People's Hospital, 399 Hailing Road, Taizhou, Jiangsu 225300, P.R. China

E-mail: yejun14718@163.com

E-mail: yuhong12311@163.com

${ }^{*}$ Contributed equally

Key words: ncRNA-ATB, ovarian cancer, proliferation, apoptosis, metastasis

\section{Introduction}

Ovarian cancer is one of the most common causes of gynecological reproductive system tumors (1). There are approximately 299,000 new cases of ovarian cancer and 152,000 related deaths each year, accounting for the seventh highest incidence among female malignant tumors (2). Most patients are already at an advanced stage by the time of diagnosis, and the 5-year survival rate of advanced ovarian cancer is less than $30 \%$ (3). Only $15 \%$ of patients are diagnosed at an early stage (stage 1) with a 5-year survival rate of $92 \%$ (4). In recent years, much research has focused on the development of new potential ovarian cancer targeted therapies (5-7).

Long non-coding RNAs (lncRNAs) are a class of RNAs with a length of more than 200 nucleotides that lack a meaningful open reading frame (8). LncRNAs participate extensively in embryonic development, apoptosis, cell cycle regulation, tumor formation and other biological processes (9-12). Previous studies have found that lncRNA plays an important role in tumor metastasis, and abnormal expression of many lncRNAs is directly related to tumor metastasis or recurrence (13-15). LncRNA activated by transforming growth factor- $\beta$ (lncRNA-ATB) is part of a family of lncRNAs, which can regulate the invasion and metastasis of various tumor cells, such as intestinal cancer and liver cancer, by regulating cell phenotype switching (16-18). However, there have been no reports on the role of IncRNA-ATB in ovarian cancer. This study therefore aimed to investigate the role of lncRNA-ATB in ovarian cancer cells and to further explore the underlying mechanisms of its action.

\section{Materials and methods}

Cell culture and treatments. Ovarian cancer cell line SKOV3 (cat no. ATCC ${ }^{\circledR}$ HTB-77 ${ }^{\mathrm{TM}}$ ) was obtained from American Type Culture Collection. Non-tumorous human ovarian surface epithelial cells (HOSEpiC; cat no. BNCC340096) were purchased from the BeNa Culture Collection (Suzhou Bei Na Chuanglian Biotechnology Co., Ltd.). SKOV3 and HOSEpiC cells were cultured in complete Dulbecco's Modified Eagle Medium (DMEM)/nutrient mixture F12 (Gibco, Thermo 
Fisher Scientific, Inc.) including 10\% FBS (Gibco; Thermo Fisher Scientific, Inc.) and $1 \%$ penicillin-streptomycin solution at $37^{\circ} \mathrm{C}$ in a $5 \% \mathrm{CO}_{2}$ incubator.

Cell transfection. LncRNA-ATB-shRNA and the negative control of lncRNA-ATB-shRNA (lncRNA-ATB-NC) were purchased from Biomics (Biomics Biotechnologies, Co., Ltd.). A total of $1 \mu \mathrm{g}$ LncRNA-ATB-NC or $1 \mu \mathrm{g}$ lncRNA-ATB-shRNA were transfected into SKOV3 cells using Lipofectamine 2000 (Invitrogen, Thermo Fisher Scientific, Inc.) according to the manufacturer's instructions. Total RNA was extracted from the cells $48 \mathrm{~h}$ after transfection, and the expression of lncRNA-ATB was detected using reverse transcription-quantitative PCR (RT-qPCR) to determine the transfection efficiency. Cells without any treatment Were considered as the control.

CCK-8 assay. The CCK- 8 method was used to determine SKOV3 cell proliferative ability. A cell suspension at a concentration of $1 \times 10^{4} / \mathrm{ml}$ was prepared and $100 \mu \mathrm{l}$ of this suspension was added to each well of a 96-well plate. Cells were then cultured for 12,24 or $48 \mathrm{~h}$, respectively, before $10 \mu \mathrm{l}$ CCK-8 reagent (Sigma-Aldrich; Merck KGaA) was added to each well. After $2 \mathrm{~h}$ of incubation absorbance at a wavelength of $450 \mathrm{~nm}$ was measured using the FLUOstar ${ }^{\circledR}$ Omega Microplate Reader (BMG Labtech $\mathrm{GmbH}$ ). This experiment was repeated three times.

Flow cytometry analysis. SKOV3 cells of different groups (control: Cells without any treatment, lncRNA-ATB-NC and lncRNA-ATB-shRNA respectively) were harvested using $0.2 \%$ trypsin, washed with phosphate buffered saline (PBS) and then fixed with $70 \%$ ethanol overnight at $4^{\circ} \mathrm{C}$. The number of apoptotic cells was quantified using the AnnexinV-FITC/PI kit (cat no. 70-AP101-100; MultiSciences) according to the manufacturer's instructions. Cell apoptosis rate was measured using a FACS Calibur flow cytometer (BD Biosciences) and the data were analyzed using FlowJo software (version 7.6.1; FlowJo LLC). The assay was performed in triplicate.

Transwell assay. A transwell invasion assay was used to determine cell invasion ability. Polycarbonate filters $(8-\mu \mathrm{m}$ pore size; Corning Inc.) with Matrigel (BD Biosciences) were used in the transwell migration assay. A total of $200 \mu \mathrm{l}$ DMEM (supplemented with $0.1 \%$ FBS) containing $1 \times 10^{5}$ SKOV3 cells was added to the upper chamber while $600 \mu 1$ DMEM supplemented with $10 \%$ FBS was added to the lower chamber. After incubation for $48 \mathrm{~h}$, any SKOV3 cells that had invaded into the lower chamber were fixed with $100 \%$ methanol at room temperature for $20 \mathrm{~min}$, stained with $0.1 \%$ crystal violet at $37^{\circ} \mathrm{C}$ for $20 \mathrm{~min}$, and counted using an upright microscope (magnification, x200; five randomly-selected fields per chamber). Each transwell assay was repeated in five independent experiments.

Scratch wound healing assay. SKOV3 cells from different treatment groups (control, lncRNA-ATB-NC and IncRNA-ATB-shRNA, respectively) were seeded onto six-well plates $\left(5 \times 10^{5}\right.$ cells/well) and cultured until they reached $80-90 \%$ confluence. Scratch wounds were created in the monolayer of confluent SKOV3 cells using a $200 \mu \mathrm{l}$ pipette tip. The wounded cells were then incubated at $37^{\circ} \mathrm{C}$ with $5 \% \mathrm{CO}_{2}$ for $24 \mathrm{~h}$. Wound healing was measured using ImageJ software version 1.46 (National Institutes of Heath) and images were captured using a phase-contrast microscope at two time points, immediately after scratching and after 24-h incubation. Data were presented as wound width (at $24 \mathrm{~h}$ )/wound width (at $0 \mathrm{~h}$ ) x100\%.

$R T-q P C R$. Total RNA was isolated from cells using anEASYspin Plus tissue/cell RNA extraction kit (Aidlab Biotechnologies, Co. Ltd.) according to the manufacturer's instructions. RNA was reverse-transcribed into cDNA using a Transcriptor First Strand cDNA Synthesis kit (Roche Diagnostics) following the manufacturer's instructions. qPCR was performed on a LightCycler 480 system (Roche Diagnostics) using Fast SYBR Green Master Mix (Roche Diagnostics) as per the manufacturer's instructions. The sequences of RT-qPCR primers were obtained as required and listed as following: GAPDH forward, 5'-CTTTGGTATCGTGGAAGGACTC-3'; reverse, 5'-GTA GAGGCAGGGATGATGTTCT-3'; lncRNA- ATB forward, 5'-TCTGGCTGAGGCTGGTTGAC-3'; reverse, 5'-ATCTCT GGGTGCTGGTGAAGG-3'; STAT3 forward, 5'-ACAGCA GGATGGCCAGGTTGC-3'; reverse, 5'-TCTGTCTGGTGG CTGCTGCCT-3'; E-Cadherin forward, 5'-AGCCATGTACGT TGCTATCC-3'; reverse, 5'-CGTAGCACAGCTTCTCCTTAA T-3'; Vimentin forward, 5'-GCTGCAGGCCCAGATTCA-3'; reverse, 5'-TTCATACTGCTGGCGCACAT-3'. The following thermocycling conditions were used for PCR: Initial denaturation at $95^{\circ} \mathrm{C}$ for $45 \mathrm{sec}$, followed by 40 cycles of $95^{\circ} \mathrm{C}$ for $10 \mathrm{sec}$ and $52^{\circ} \mathrm{C}$ for $35 \mathrm{sec}$. The relative expression ratio of target genes was calculated using the $2^{-\Delta \Delta C q}$ method (19).

Western blotting. Cells were washed twice with PBS, then lysed in RIPA buffer (cat. no. P0013B; Beyotime Institute of Biotechnology). Protein concentration was measured using a BCA assay kit (Thermo Fisher Scientific, Inc.). The cell lysates (30 $\mu \mathrm{g} /$ lane) were separated on $12 \%$ SDS polyacrylamide gels and transferred onto PVDF membranes (Bio-Rad Laboratory, Inc.). After blocking of non-specific binding with TBS-T ( $0.1 \%$ Tween) containing 5\% non-fat milk for $1 \mathrm{~h}$ at room temperature, the membranes were incubated with the primary antibodies at $4^{\circ} \mathrm{C}$ overnight. Then, the membranes were incubated with horseradish peroxidase (HRP)-conjugated goat anti-rabbit secondary antibody (cat no. 7074; 1:2,000; Cell Signaling Technology, Inc.) for $2 \mathrm{~h}$ at room temperature. Finally, the protein bands were detected using the ChemiDOC $^{\text {TM }}$ system (Bio-Rad, Hercules, CA) following the manufacturer's protocol. The primary antibodies: Anti-STAT3 (1:1,000; cat no. 12640), anti-p-STAT3 (1:1,000; cat no. 9145), anti-E-cadherin (1:1,000; cat no. 3195), anti-vimentin (1:1,000; cat no. 5741) and anti-GAPDH (cat no. 5174) were purchased from Cell Signaling Technology, Inc. Protein bands were analyzed using ImageJ version 1.49 software (National Institute of Health).

Statistical analysis. Statistical analysis was performed using GraphPad Prism version 5.0 (GraphPad Software, Inc.). The tests performed were one-way ANOVA followed by Tukey's post-hoc test or a Student's t-test as appropriate. Data are presented as the mean $\pm S D$. $P<0.05$ was considered to indicate a statistically significant difference. 
A

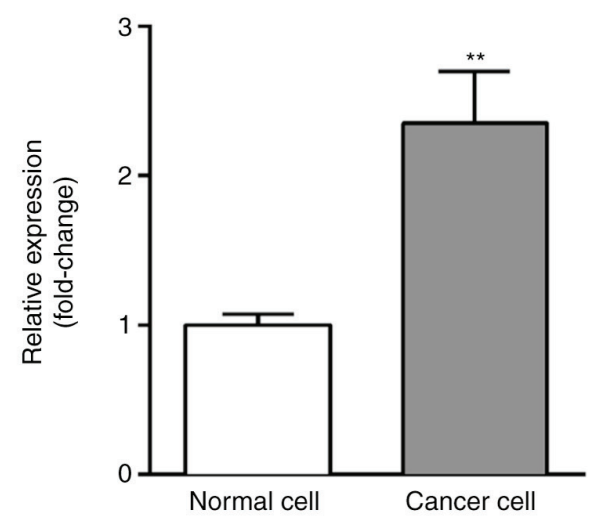

$\mathrm{B}$

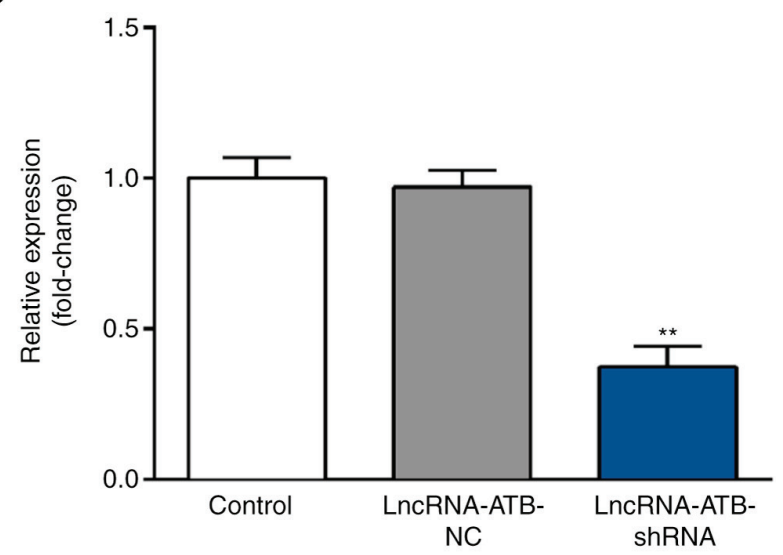

Figure 1. Expression of lncRNA-ATB in ovarian cancer cell line SKOV3. (A) RT-qPCR was used to detect the levels of lncRNA-ATB in SKOV3 cells and HOSEpiCs. (B) LncRNA-ATB-NC or lncRNA-ATB-shRNA was transfected into SKOV3 cells for 48 h, and the expression of lncRNA-ATB in SKOV3 cells was detected by RT-qPCR. Data were presented as the mean \pm SD. Each experiment was repeated three times. ${ }^{* *} \mathrm{P}<0.01$ vs. control group. HOSEpiC, human ovarian surface epithelial cell; lncRNA-ATB, long non-coding RNA-activated by transforming growth factor- $\beta$; lncRNA-ATB-NC, long non-coding RNA-activated by transforming growth factor- $\beta$-negative control; RT-qPCR, reverse transcription-quantitative polymerase chain reaction; SKOV3, ovarian cancer cell line.

\section{Results}

LncRNA-ATB is highly expressed in ovarian cancer cell line SKOV3. Expression levels of 1ncRNA-ATB in SKOV3 and HOSEpiC cells were measured using RT-qPCR. The results indicated that when compared with HOSEpiC cells, lncRNA-ATB was significantly upregulated in SKOV3 cells (Fig. 1A). To further investigate the role of lncRNA-ATB in ovarian cancer, lncRNA-ATB-shRNA or lncRNA-ATB-NC were transfected into SKOV3 cells. RT-qPCR results revealed that compared with the control group, IncRNA-ATB-shRNA significantly decreased the expression of lncRNA-ATB in SKOV3 cells (Fig. 1B).

LncRNA-ATB downregulation reduces proliferation and induces apoptosis in SKOV3 cells. A CCK-8 assay was used to analyze cell proliferation and flow cytometry was used to analyze apoptosis. The results revealed that when compared with the control group, lncRNA-ATB-shRNA significantly reduced the proliferative ability of SKOV3 cells at the 24 and $48 \mathrm{~h}$ time points (Fig. 2A) and promoted apoptosis (Fig. 2B).

LncRNA-ATB downregulation reduces migration and invasion of SKOV3 cells. To study the effect of IncRNA-ATB downregulation on ovarian cancer cell migration and invasion, scratch wound healing and transwell assays were performed. The results revealed that when compared with the control group, IncRNA-ATB-shRNA significantly reduced the migration (Fig. 3A) and invasion ability (Fig. 3B) of SKOV3 cells.

LncRNA-ATB downregulation significantly reduces the phosphorylation of STAT3, increases E-cadherin expression and decreases vimentin expression in SKOV3 cells. The effect of lncRNA-ATB downregulation on ovarian cancer cell epithelial to mesenchymal transition (EMT) was examined through analysis of the expression of EMT-related proteins (E-cadherin and vimentin) in SKOV3 cells. Transfection with lncRNA-ATB-shRNA significantly increased E-cadherin protein and mRNA expression (Fig 4A, B and D), and decreased vimentin protein and mRNA expression (Fig. 4A, $\mathrm{B}$ and $\mathrm{E})$.

Finally, to explore the molecular mechanisms behind the role of lncRNA-ATB in ovarian cancer, the STAT3 pathway was studied via analysis of the phosphorylation of STAT3 using western blotting. The results suggested that lncRNA-ATB-shRNA significantly inhibited the phosphorylation of STAT3 (Fig. 4A and B), but had no significant effect on STAT3 expression (Fig. 4A and C).

\section{Discussion}

Ovariancancer, the most lethal of all gynecological malignancies, ranks as the fifth leading cause of cancer deaths in women $(1,2)$. The prognosis of ovarian cancer is generally poor due to the lack of clear symptoms and effective screening and diagnostic methods, especially for identifying early stages of the disease. For this reason the identification of better tumor bio-markers to predict the prognosis of ovarian cancer has attracted the attention of oncologists (20-25). Increasing evidence suggests that lncRNAs play vital roles in tumorigenesis and the invasion and metastasis of cancer (26-28). Several studies have evaluated the prognostic value of IncRNA-ATB expression in human cancer $(29,30)$. These previous studies (26-30) indicated that lncRNA-ATB may serve important roles in ovarian cancer. In the present study, IncRNA-ATB was significantly upregulated in ovarian cancer cells compared with healthy ovarian cells, suggesting the possible involvement of lncRNA-ATB in ovarian cancer development. Furthermore, IncRNA-ATB downregulation effectively reduced the proliferative, invasive and migratory capabilities of ovarian cancer cells and significantly increased apoptosis. These data indicate that lncRNA-ATB may promote cell proliferation, invasion and migration, and inhibit cell apoptosis in ovarian cancer cells.

The discovery of EMT, as a mechanism that allows cancer cells to dedifferentiate and acquire enhanced migratory and invasive properties, has had a significant impact on cancer 
A

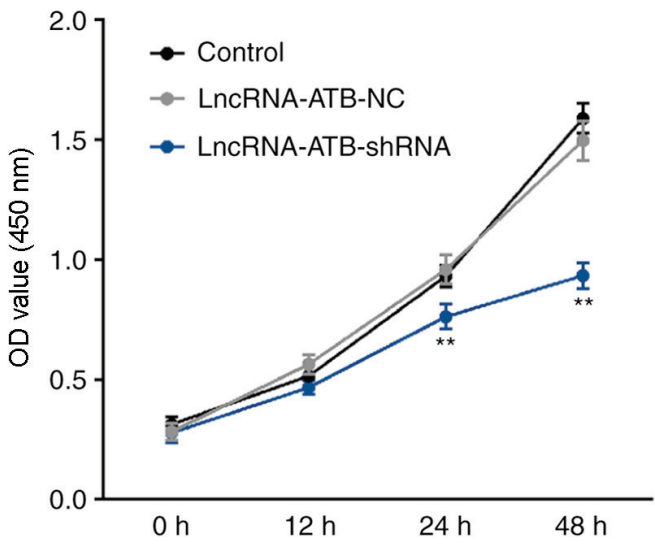

B

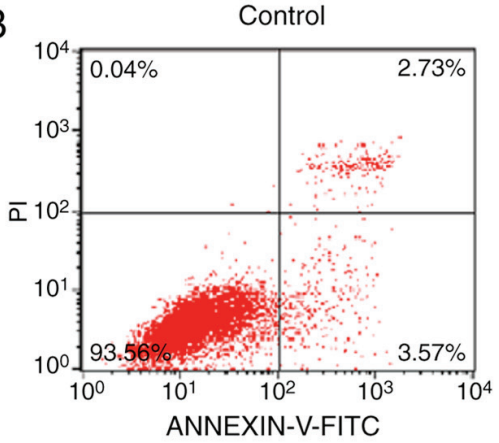

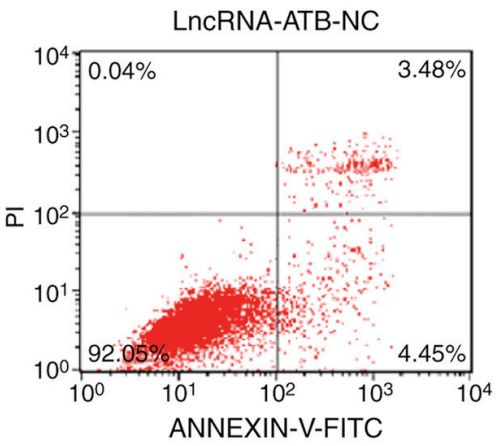

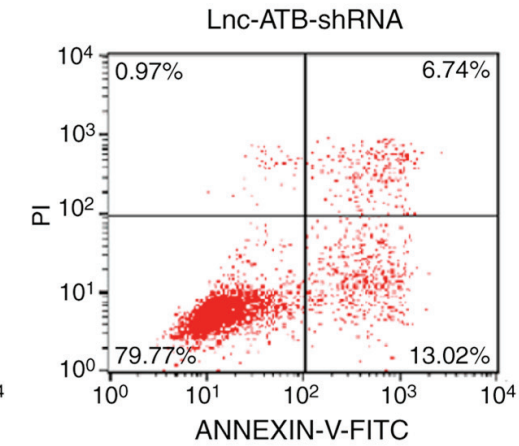

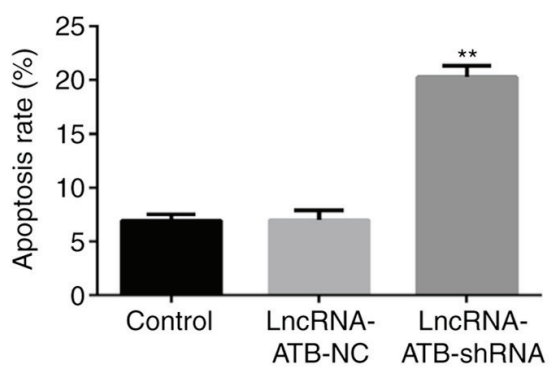

Figure 2. Effect of lncRNA-ATB downregulation on SKOV3 cell proliferation and apoptosis. LncRNA-ATB-NC or lncRNA-ATB-shRNA was transfected into SKOV3 cells for $48 \mathrm{~h}$. (A) Cell counting kit-8 assay was used to detect cell proliferation ability. (B) Flow cytometry was used to detect the apoptosis level. Data are presented as the mean \pm SD. Each experiment was repeated three times. ${ }^{* *} \mathrm{P}<0.01$ vs. control group. LncRNA-ATB, long non-coding RNA-activated by transforming growth factor- $\beta$; lncRNA-ATB-NC, long non-coding RNA-activated by transforming growth factor- $\beta$-negative control; SKOV3, ovarian cancer cell line; PI, propidium iodide.

research $(31,32)$. EMT describes the process whereby epithelial cells undergo multiple biological changes and transform into interstitial tissues with strongly invasive characteristics $(33,34)$. Current research indicates that there are three types of EMT, EMT associated with embryo implantation, EMT associated with tissue fibrosis and remodeling characteristics, and EMT associated with strong carcinogenic effects (35). Tumor cells contribute to the formation of fibrosis through the EMT process and affect the secretion pattern of cytokines to promote tumorigenesis (36). Moreover, in EMT, initially inactive polarized epithelial cells dissolve their own intercellular junctions to form independent, depolarized and active metastatic mesenchymal cells $(37,38)$. For example, the expression and function of E-cadherin linked to epithelial cells can be lost, while $\mathrm{N}$-cadherin, which induces adhesion to mesenchymal cells is induced (39). EMT is an important process for cancer cell migration and metastasis and can be induced by various transcription factors and signal transduction factors (37-39). In the current study, the effect of lncRNA-ATB downregulation on ovarian cancer cell EMT was examined through assessment of the expression of EMT-related proteins (E-cadherin and vimentin) in SKOV3 cells. This study showed that lncRNA-ATB downregulation may inhibit ovarian cancer cell EMT.

STAT3 enhances various signaling pathways involved in the regulation of cell growth, invasion and EMT, and is recognized as a key target for cancer treatment (40-42). The STAT3 signaling pathway plays an important role in ovarian cancer progression (43-45). In order to explore the molecular mechanism behind the role of lncRNA-ATB in ovarian cancer, the STAT3 pathway was analyzed. IncRNA-ATBA downregulation significantly reduced STAT3 activation, indicating that lncRNA-ATB may act on ovarian cancer cells at least partly through influencing the STAT3 signaling pathway activation.

In conclusion, this study demonstrated that lncRNA-ATB was highly expressed in ovarian cancer cells, and that its 

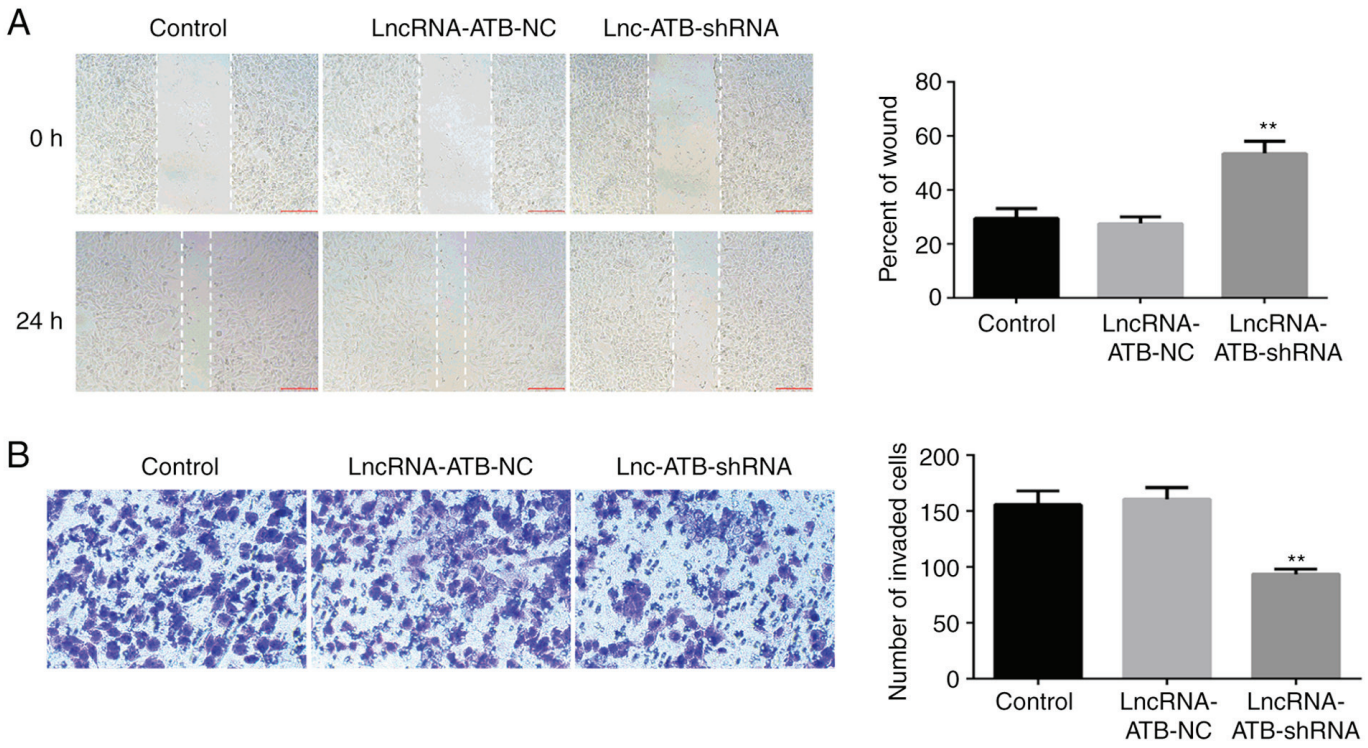

Figure 3. Effects of lncRNA-ATB downregulation on SKOV3 cell invasion and migration. (A) LncRNA-ATB-NC or lncRNA-ATB-shRNA was transfected into SKOV3 cells for $48 \mathrm{~h}$, then scratch wound healing assay was used to detect cell migration ability. (B) Transwell assay was used to detect cell invasion ability (magnification, $\mathrm{x} 200$ ). Data are presented as the mean $\pm \mathrm{SD} .{ }^{* *} \mathrm{P}<0.01$ vs. control group. IncRNA-ATB, long non-coding RNA-activated by transforming growth factor- $\beta$; lncRNA-ATB-NC, long non-coding RNA-activated by transforming growth factor- $\beta$-negative control; SKOV3, ovarian cancer cell line.

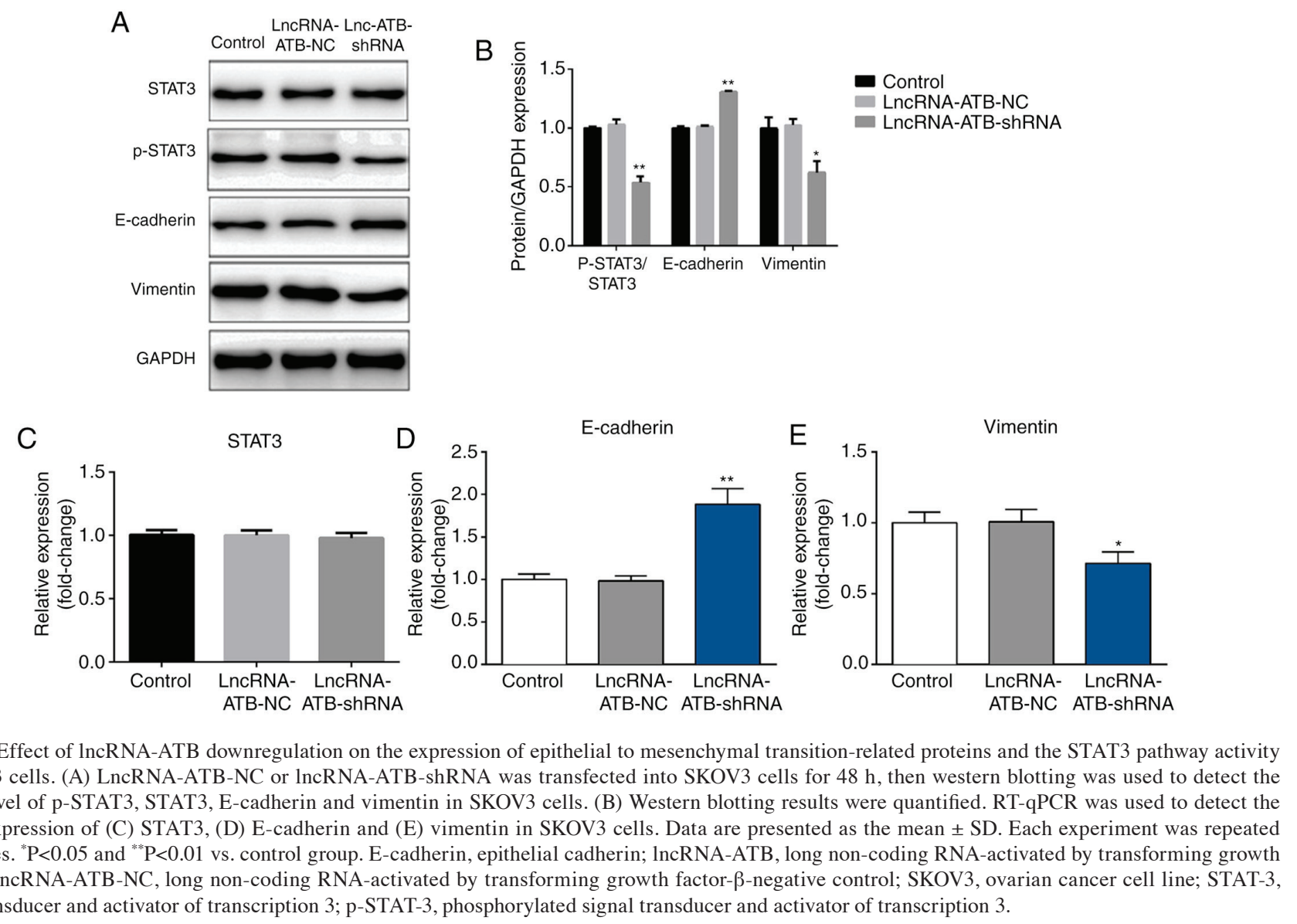

downregulation effectively inhibited ovarian cancer cell proliferation, invasion, and migration and induced cell apoptosis. These effects might be mediated by the regulation of the STAT3 signaling pathway. Therefore, lncRNA-ATB may be a new therapeutic target for the treatment of ovarian cancer.
However, this study only includes a preliminary analysis. To clarify the role of lncRNA-ATB in ovarian cancer, further research is needed. The role of IncRNA-ATB in other ovarian cancer cell lines and in ovarian cancer in vivo should be studied. These issues will be addressed in future studies. 


\section{Acknowledgements}

Not applicable.

\section{Funding}

No funding was received.

\section{Availability of data and materials}

All data sets used and/or generated during the current study are available from the corresponding author on reasonable request.

\section{Authors' contributions}

DY and XZ contributed to study design, data collection, statistical analysis, data interpretation and manuscript preparation. YZ, HQ, HW, CH, XL, TG and ML contributed to data collection and statistical analysis. HY and JY contributed to study design, data interpretation and manuscript preparation.

\section{Ethics approval and consent to participate}

Not applicable.

\section{Patient consent for publication}

Not applicable.

\section{Competing interests}

The authors declare that they have no competing interests.

\section{References}

1. Webb PM and Jordan SJ: Epidemiology of epithelial ovarian cancer. Best Pract Res Clin Obstet Gynaecol 41: 3-14, 2017.

2. Reid BM, Permuth JB and Sellers TA: Epidemiology of ovarian cancer: A review. Cancer Biol Med 14: 9-32, 2017.

3. Pierredon S, Ribaux P, Tille JC, Petignat P and Cohen M: Comparative secretome of ovarian serous carcinoma: Gelsolin in the spotlight. Oncol Lett 13: 4965-4973, 2017.

4. Howlader N, Noone A, Krapcho M, Neyman N, Aminou R, Waldron W, Altekruse SF, Kosary CL, Ruhl J, Tatalovich Z et al: SEER Cancer Statistics Review, 1975-2008, National Cancer Institute.

5. Grunewald T and Ledermann JA: Targeted Therapies for Ovarian Cancer. Best Pract Res Clin Obstet Gynaecol 41: 139-152, 2017.

6. Kim JY, Cho CH and Song HS: Targeted therapy of ovarian cancer including immune check point inhibitor. Korean J Intern Med 32: 798-804, 2017.

7. Brasseur K, Gévry N and Asselin E: Chemoresistance and targeted therapies in ovarian and endometrial cancers. Oncotarget 8: 4008-4042, 2017.

8. Lintner NG and Cate J: Regulating the ribosome: A spotlight on RNA dark matter. Mol Cell 54: 1-2, 2014.

9. Ponting CP, Oliver PL and Rei W: Evolution and functions of long noncoding RNAs. Cell 136: 629-641, 2009.

10. Cesana M, Cacchiarelli D, Legnini I, Santini T, Sthandier O, Chinappi M, Tramontano A and Bozzoni I: A long noncoding RNA controls muscle differentiation by functioning as a competing endogenous RNA. Cell 147: 358-369, 2011.

11. Kitagawa M, Kitagawa K, Kotake Y, Niida $\mathrm{H}$ and Ohhata T: Cell cycle regulation by long non-coding RNAs. Cell Mol Life Sci 70: 4785-4794, 2013.

12. Zhang XM, Yang GL, Le XH, et al: Expression profiles of long non-coding RNA in HCC patients suffering hepatitis B liver cirrhosis. J Zhengzhou Univ (Med Sci) 50: 194-198, 2015.
13. Yu T, Zhao Y, Hu Z, Li J, Chu D, Zhang J, Li Z, Chen B, Zhang X, Pan H, et al: MetaLnc9 facilitates lung cancer metastasis via a PGK1-activated AKT/ mTOR pathway. Cancer Res 77: 5782-5794, 2017.

14. Su W, Xu M, Chen X, Chen N, Gong J, Nie L, Li L, Li X, Zhang M and Zhou Q: Long noncoding RNA ZEB1-AS1 epigenetically regulates the expressions of ZEB1 and downstream molecules in prostate cancer. Mol Cancer 16: 142, 2017.

15. Dey BK, Mueller AC and Dutta A: Long non-coding RNAs as emerging regulators of differentiation, development, and disease. Transcription 5: e944014, 2014.

16. Iguchi T, Uchi R, Nambara S, Saito T, Komatsu H, Hirata H, Ueda M, Sakimura S, Takano Y, Kurashige J, et al: A long noncoding RNA, lncRNA-ATB, is involved in the progression and prognosis of colorectal cancer. Anticancer Res 35: 1385-1388, 2015.

17. Fu N, Zhao SX, Kong LB, Du JH, Ren WG, Han F, Zhang QS, Li WC, Cui P, Wang RQ, et al: LncRNA-ATB/microRNA-200a/ $\beta$-catenin regulatory axis involved in the progression of HCV-related hepatic fibrosis. Gene 618: 1-7, 2017.

18. Ke L, Xu SB, Wang J, Jiang XL and Xu MQ: High expression of long non-coding RNA ATB indicates a poor prognosis and regulates cell proliferation and metastasis in non-small cell lung cancer. Clin Transl Oncol 19: 599-605, 2017.

19. Livak KJ and Schmittgen TD: Analysis of relative gene expression data using real-time quantitative PCR and the 2(-Delta Delta $\mathrm{C}(\mathrm{T})$ ) method. Methods 25: 402-408, 2001.

20. Chen S, Zhang L, Yan G, Cheng S, Fathy AH, Yan N and Zhao Y: Neutrophil-to-lymphocyte ratio is a potential prognostic biomarker in patients with ovarian cancer: A Meta-analysis. Biomed Res Int 2017: 7943467, 2014.

21. Wang J, Zhao Y, Qi R, Zhu X, Huang C, Cheng S, Wang S and Qi X: Prognostic role of podocalyxin-like protein expression in various cancers: A systematic review and meta-analysis. Oncotarget 8: 52457-52464, 2017.

22. Zhou Y, Cheng S, Chen S and Zhao Y: Prognostic and clinicopathological value of SIRT3 expression in various cancers: A systematic review and meta-analysis. OncoTargets Ther 11: 2157-2167, 2018

23. Zhou Y, Cheng S, Fathy AH, Qian H and Zhao Y: Prognostic value of platelet-tolymphocyte ratio in pancreatic cancer: A comprehensive meta-analysis of 17 cohort studies. OncoTargets Ther 11: 1899-1908, 2018.

24. Zhao Y, Si G, Zhu F, Hui J, Cai S, Huang C, Cheng S, Fathy AH, Xiang Y and Li J: Prognostic role of platelet to lymphocyte ratio in hepatocellular carcinoma: A systematic review and meta-analysis. Oncotarget 8: 22854-22862, 2017.

25. Song X, Yao H, Liu J and Wang Q: The prognostic value of long noncoding RNA Sox 2 ot expression in various cancers: A systematic review and meta-analysis. Clin Chim Acta 484: 52-59, 2018.

26. Hu Y, Chen HY, Yu CY, Xu J, Wang JL, Qian J, Zhang X and Fang JY: A long non-coding RNA signature to improve prognosis prediction of colorectal cancer. Oncotarget 5: 2230-2242, 2014.

27. Beckedorff FC, Amaral MS, Deocesano-Pereira C and Verjovski-Almeida S: Long non-coding RNAs and their implications in cancer epigenetics. Biosci Rep 33: pii: e00061, 2013.

28. Gutschner T and Diederichs S: The hallmarks of cancer: A long non-coding RNA point of view. RNA Biol 9: 703-719, 2012.

29. Chen Y, Wei G, Xia H, Tang Q and Bi F: Long noncoding RNAATB promotes cell proliferation, migration and invasion in gastric cancer. Mol Med Rep 17: 1940-1946, 2018.

30. Fu XM, Guo W, Li N, Liu HZ, Liu J, Qiu SQ, Zhang Q, Wang LC, $\mathrm{Li} \mathrm{F}$ and Li CL: The expression and function of long noncoding RNA IncRNA-ATB in papillary thyroid cancer. Eur Rev Med Pharmacol Sci 21: 3239-3246, 2017.

31. Karlsson MC, Gonzalez SF, Welin J and Fuxe J: Epithelial-mesenchymal transition in cancer metastasis through the lymphatic system. Mol Oncol 11: 781-791, 2017.

32. Liao TT and Yang MH: Revisiting epithelial-mesenchymal transition in cancer metastasis: The connection between epithelial plasticity and stemness. Mol Oncol 11: 792-804, 2017.

33. Davis FM, Azimi I, Faville RA, Peters AA, Jalink K, Putney JW Jr, Goodhill GJ, Thompson EW, Roberts-Thomson SJ, Monteith GR, et al: Induction of epithelial-mesenchymal transition (EMT) in breast cancer cells is calcium signal dependent. Oncogene 33: 2307-2316, 2014. 
34. Yoshida T, Ozawa Y, Kimura T, Sato Y, Kuznetsov G, Xu S, Uesugi M, Agoulnik S, Taylor N, Funahashi Y and Matsui J: Eribulin mesilate suppresses experimental metastasis of breast cancer cells by reversing phenotype from epithelial-mesenchymal transition (EMT) to mesenchymal-epithelial transition (MET) states. Br J Cancer 110: 1497-1505, 2014.

35. Qu C, Zhang W, Zheng G, Zhang Z, Yin J and He Z: Metformin reverses multidrug resistance and epithelial-mesenchymal transition (EMT) via activating AMP-activated protein kinase (AMPK) in human breast cancer cells. Mol Cell Biochem 386: 63-71, 2014.

36. Vetuschi A, Pompili S, Flati V, Berardinis LD, Di Gregorio J, Latella G and Sferra R: Epithelial-to-Mesenchimal Transition (EMT) in experimental intestinal fibrosis. Italian J Anatomy Embryology 120: 58, 2015.

37. Yang Y, Gao M, Lin Z, Chen L, Jin Y, Zhu G, Wang Y and Jin T: DEK promoted EMT and angiogenesis through regulating $\mathrm{PI} 3 \mathrm{~K} / \mathrm{AKT} / \mathrm{mTOR}$ pathway in triple-negative breast cancer. Oncotarget 8: 98708-98722, 2017.

38. Ma Z, Xin Z, Hu W, Jiang S, Yang Z, Yan X, Li X, Yang Y and Chen F: Forkhead box O proteins: Crucial regulators of cancer EMT. Semin Cancer Biol 50: 21-31, 2018.

39. Zuo L, Zhao H, Yang R, Wang L, Ma H, Xu X, Zhou P and Kong L: Lamin A/C might be involved in the EMT signalling pathway. Gene 663: 51-64, 2018.
40. Zhao J, Du P, Cui P, Qin Y, Hu C, Wu J, Zhou Z, Zhang W, Qin L and Huang G: LncRNA PVT1 promotes angiogenesis via activating the STAT3/VEGFA axis in gastric cancer. Oncogene 37: 4094-4109, 2018

41. Egusquiaguirre SP, Yeh JE, Walker SR, Liu S and Frank DA: The STAT3 Target Gene TNFRSF1A Modulates the NF- $\kappa$ B Pathway in Breast Cancer Cells. Neoplasia 20: 489-498, 2018.

42. Bournazou E and Bromberg J: Targeting the tumor microenvironment: JAK-STAT3 signaling. JAKSTAT 2: e23828, 2013.

43. Chen L, Wang J, Wu J, Zheng Q and Hu J: Indirubin suppresses ovarian cancer cell viabilities through the STAT3 signaling pathway. Drug Des Devel Ther 12: 3335-3342, 2018.

44. Jia ZH, Jia Y, Guo FJ, Chen J, Zhang XW and Cui MH: Phosphorylation of STAT3 at Tyr705 regulates MMP-9 production in epithelial ovarian cancer. PLoS One 12: e0183622, 2017.

45. Saini U, Naidu S, ElNaggar AC, Bid HK, Wallbillich JJ, Bixel K, Bolyard C, Suarez AA, Kaur B, Kuppusamy P, et al: Elevated STAT3 expression in ovarian cancer ascites promotes invasion and metastasis: A potential therapeutic target. Oncogene 36: 168-181, 2017.

(i) () $($ This work is licensed under a Creative Commons International (CC BY-NC-ND 4.0) License. 